\title{
Additional clinical sign of "unilateral" endocrine exophthalmos
}

\author{
JITSCHAK HAUER \\ Jaffa, Israel
}

There may be difficulties in the diagnosis of unilateral endocrine exophthalmos particularly in the differential diagnosis from orbital tumour.

It is the purpose of this communication to show that endocrine ophthalmopathy i i always bilateral, although its exophthalmic component is frequently unilateral in its earliw stages. This observation could be the basis of differential diagnosis between "unilateraP endocrine exophthalmos" and retrobulbar tumour.

To facilitate differential diagnosis, use is made of a phenomenon already reported b $\vec{\psi}$ Birch-Hirschfeld (1930) that in all normal subjects there is a difference in the exophthal mometric readings in the upright and recumbent position. In all normal subjects the globe sinks back when the patient is recumbent, and the exophthalmometric reading i reduced by i to $3 \mathrm{~mm}$.

Hauer (1956) has shown that this postural difference is absent in both eyes in cases of endocrine ophthalmopathy and that this is independent of the presence or absence of visible exophthalmos. Thus, the presence of the physiological postural difference in thळ opposite side in a case of orbital tumour and its absence in a case of unilateral endocrin命 exophthalmos may be used as the basis in the differential diagnosis between the two con $\vec{F}$ ditions. This is illustrated in the following cases, where the assumption that endocrine exophthalmos is, even in its initial unilateral phase as judged by conventional methods a bilateral condition, while orbital tumours are usually unilateral, assisted the correct: diagnosis.

\section{Case reports}

(1) A 55-year-old woman presented with an exophthalmos of $17 \mathrm{~mm}$. in the right eye as compared with $14 \mathrm{~mm}$. in the left. Sursumversion of the right eye was defective. The movements of the left eye were normal. General examination failed to reveal signs of endocrine disorder, and ne radiological evidence of a retrobulbar tumour was found. Neither eye showed reduction of the exophthalmometric reading when the patient was in the recumbent position. Nevertheless, surgic ân exploration of the right orbit had been carried out elsewhere and found to be negative. ExophN thalmos and ophthalmoplegia developed in the left eye 3 years later and the diagnosis of endocrin£ ophthalmopathy was clear.

(2) A 57-year-old man showed exophthalmos of $21 \mathrm{~mm}$. in the right eye, the reading in the lef eye being $15 \mathrm{~mm}$. The right eye was proptosed and dislocated outwards and downwards and there was impaired sursumversion. The optic nerve was not abnormal in appearance or function There was no sign of endocrine disturbance, and no radiological evidence of a retrobulba婴 
tumour. Neither eye showed reduction of the exophthalmometric reading when the patient was in the recumbent position and it was, therefore, decided to wait. One year later the second eye showed exophthalmic ophthalmoplegia, and there was no longer any doubt about the endocrine basis of the condition.

(3) and (4) After the introduction of this method of differential diagnosis, two adults aged 32 and 40 years with retrobulbar tumour diagnosed radiologically were hospitalized in this department. Both patients were operated on and the histological reports gave the diagnosis of endothelioma. In each case the globe sank back on the healthy side when the patient was in the recumbent position but did not sink on the side of the tumour.

\section{Comment}

In Cases $I$ and 2 the diagnosis of endocrine exophthalmos was supported by the fact that the apparently unaffected eye did not sink back into the orbit when the patient was recumbent.

In Cases 3 and 4, however, the normal regression of the globe on the side unaffected by the tumour supported the diagnosis of retrobulbar neoplasm.

\section{Summary}

In cases of "unilateral" exophthalmos due to endocrine disturbance the physiological postural difference in the unaffected eye is absent, but in cases of unilateral orbital tumour this postural difference is present. This may be used as a method of differential diagnosis to avoid unnecessary surgical exploration in cases of endocrine exophthalmos.

\section{References}

BIRCH-HIRSCHFeld, A. (1930) In “Graefe-Saemisch Handbuch der gesamten Augenheilkunde", 2nd ed. band 9, abt. I, teil I, p. 22. Springer, Berlin

HAUER, J. (1956) Brit. F. Ophthal., 40, 553 